\title{
Nursing Students' Perceptions, Beliefs and Attitudes: A First Study on Special Pedagogic Activities
}

\author{
Ioannidi Vassiliki \\ $\mathrm{BSc}, \mathrm{PhD}$, University of Peloponnese \\ Faculty of Human Movement and Quality of Life Sciences \\ Department of Nursing, 6SDW Greece \\ E-mail: vioannid@uop.gr \\ Kolovos Petros \\ $\mathrm{RN}, \mathrm{BSc}, \mathrm{MSc}, \mathrm{PhD}(\mathrm{c})$, University of Peloponnese \\ Faculty of Human Movement and Quality of Life Sciences \\ Department of Nursing, 6SDW Greece \\ E-mail:pkolovos@uop.gr \\ Vasileiadou Sofia \\ RN, BSc, MSc, General University Hospital "Attikon" \\ Sparti, Greece \\ E-mail: sofvasileiadou@gmail.com \\ Malliarou Maria (Corresponding author) \\ Captain RN, MSc, PhDc, Scientific collaborator Technological Institution of Larisa \\ Nursing Department, Sparti, Greece
}

Tel: 30-241-085-1742Ｅ-mail: mmalliarou@gmail.com

Travlos K. Antonios

BSc, PhD, Assistant Professor, University of Peloponnese

Faculty of Human Movement and Quality of Life Sciences

Department of Sport Management, Sparti, Greece

E-mail: atravlos@uop.gr

Zyga Sofia

$\mathrm{RN}, \mathrm{BSc}, \mathrm{MSc}, \mathrm{PhD}$, Assistant Professor, University of Peloponnese

Faculty of Human Movement and Quality of Life Sciences

Department of Nursing, Sparti, Greece

E-mail: zygas@uop.gr

Received: November 30, 2010 Accepted: December 14, 2010 doi:10.5539/gjhs.v3n1p40 


\section{Abstract}

Background: Studying nursing students' opinions could be a useful guide for the formation of their professional role as well as their education and training.

Aim: The present study's aim is to examine nurse students' opinion, beliefs and attitude regarding the concepts of health, health care, health education, health ethics, illness, safety in health care and quality in health care, in an educational environment which promotes cooperative activities in class.

Methodology: Seventy four nurse students of the University of Peloponnese participated in the present study during the winter semester of 2008-2009. They were asked to describe shortly the meaning of the study concepts, writing down their opinion on a questionnaire that included six questions. Their answers were evaluated using the method of content analysis and sorted to exhaustive and mutually exclusive categories.

Results: Nursing Students were asked to describe the meaning of the concept of health, health care, health education, health ethics, illness, safety in health care and quality in health care. The result of the students' opinion reveals the way they conceive their professional role, their education and their attitude in real working conditions and, therefore, the quality of the services they provide and how well prepared they are to demand and to put in a claim for the quality in health care.

Conclusions: Further empirical research could aim to replicate or contradict these findings, using a larger sample and recruiting more university departments.

Keywords: Health, Care, Health education, Ethics, Illness, Safety, Quality

\section{Introduction}

Nursing Students' opinion on fundamental health parameters while practicing health care, defines their professional identity. That is the reason why studying nursing students' opinions could be a useful guide for the formation of their professional role as well as their education and training. As Kotrotsiou et al. (2008) have written, 'a person's opinion about care and the importance someone attributes to it, can affect strategies in planning continuous and individualized care'.

Consequently, it is quite important to point out that the improvement of learning procedures is directly connected to the development of Educational Sciences and, also, to the introduction of new teaching strategies in the basic core of the learning procedures. This fact forms a new interdisciplinary reality in education, which affects the learning process in health sciences education by creating new learning goals (Williams \& Taylor, 2008).

Our pedagogic intervention consists in stimulating nursing students of the University of Peloponnese to the concepts of 'health', 'health care', 'health education', 'health ethics', 'illness', 'safety in health care' and 'quality in health care' through special cooperative pedagogical activities. These activities aim at strengthening a participating environment in the classroom.

The statement of every student's personal view on the semantic of the concepts under study is a critical pedagogic parameter in founding Nurse Education. According to that, the present study's aim is to examine nurse students' opinion, beliefs and attitude regarding the concepts of 'health', 'health care', 'health education', 'health ethics', 'illness', 'safety in health care' and 'quality in health care' in an educational environment which promotes cooperative activities in class.

The expected learning outcome for these cooperative activities is the improvement in organizing multidimensional scientific activities and the empowerment of an incorporative pedagogic environment that ensure maximum active participation for the students through dialogue and interventions in their learning procedure.

\section{Aim}

The statement of every student's personal view on the semantic of the under study concepts is a critical pedagogic parameter in founding Nurse Education. According to that, the study's aim is to examine nurse students' opinion, beliefs and attitude regarding the concepts of 'health', 'health care', 'health education', 'health ethics', 'illness', 'safety in health care' and 'quality in health care', in an educational environment which promotes cooperative activities in class.

The expected learning outcome for these cooperative activities is the improvement in organizing multidimensional scientific activities and the empowerment of an incorporative pedagogic environment that ensure maximum active participation for the students through dialogue and interventions in their learning procedure. 


\section{Methodology}

\subsection{Participants}

Seventy four nursing students of the University of Peloponnese participated in the research during the winter semester of 2008-2009.

\subsection{Ethics}

The study was approved by the Ethics Committee of Nursing Department of University of Peloponnese.

Potential subjects were approached by the researchers, were given an explanation of the purpose and aims of the study, including the issue of confidentiality and they were asked to participate on a voluntary basis. An informed consent was obtained from those who agreed to participate and they were asked to complete the questionnaire.

\subsection{Data collection}

They were asked to describe shortly the meaning of the concepts 'health', 'health care', 'health education', 'health ethics', 'illness', 'safety in health care' and 'quality in health care', write down their opinion on a questionnaire that included six questions. The questionnaires were given to all the students during the winter semester of 2008-2009.

\subsection{Data analysis}

Their answers were evaluated using the method of context analysis. Context analysis' certain procedure and principles were followed (Ryan \& Bernard, 2000).

The analysis was based on the latent context (Graneheim \& Lundman, 2004) of the students' answers. The texts, which were selected as the unit of analysis, were shortened by abstracting them, which is by translating the text on a higher logical level keeping the underlying meaning of the text, in order to create codes and, after that, categories (Graneheim \& Lundman, 2004). The categories were discussed and created by the researchers following the rule that data do not fit into two categories or cannot be excluded of the present categories. In other words, the categories are exhaustive and mutually exclusive (Vamvoukas, 1988, Graneheim \& Lundman, 2004). Each of the concepts under study forms the three themes 'health', 'health care', 'health education', 'health ethics', 'illness', 'safety in health care' and 'quality in health care', which consist the categories mentioned above.

\section{Results}

The results of the texts' context analysis are presented and commented and the categories' context is given, while certain passages from the texts are also cited below.

\subsection{The concept of 'health'}

Nursing students were asked to write down on the questionnaire their opinion over the meaning of 'health', answering the question what is the meaning of 'health' in your opinion?. The answers were analysed, coded and sorted into three categories as described below:

4.1.1 The concept of 'health' as "physical, emotional, intelligence and social well being

Nursing students describe the concept of 'health' as the status in which body, soul and mind are in stability. For example,

Health is to take care of and promote physical, emotional, mental and social well being

It is the good physical, emotional and mental status of the individual

It is a situation in which someone is functional and experiences mental, social, emotional and physical well being

When someone is physically and emotionally imbalanced

The good function of emotion and body.

4.1.2 The concept of 'health' as 'absence of illness'

Nursing students also describe "health" as "the absence of illness or disability".

For example,

A situation where there is no illness or disorder

The fact that someone is not ill, has no physical or emotional illness 
Living life without experiencing illness or emotional disorder

Absence of illness and emotional disorder.

4.1.3 The concept of 'health' as 'a value of life'

Nurse students described health as the "greatest value of life" which must be protected by the individual and the community as a whole.

For example,

Health is a gift of life. Only when someone is healthy, can he enjoy life and confront with other difficulties

Health is very important as it is has a major role on how someone understands and deals with every day life situations'

Health is the goal of people and protecting it brings happiness.

4.2 The concept of 'health education'

Nursing students were asked to describe shortly the meaning of 'health education'.

4.2.1 The concept of 'health education' as 'health professionals' education and training'

Nursing students gave to 'health education' the meaning of the professional education and training of the scientists and professionals who work in health care services. The professionals' education includes knowledge, skills, values, such as respect for the patient, and ethics. Moreover, students mentioned several times the important role of continuing education for the scientists and their participation to research. For example,

Health education is about learning and gaining abilities in order to serve in the field of nursing

It means professionals who are educated, conscientious and have good knowledge, ethics, and respect

It means training someone so that he is sufficient to provide medical care

It is the theoretical and technical knowledge and values to health professionals

It involves professors and health students and participation of the last ones to do research on their science

Scientific education and ethics for health professionals. It also includes knowledge, ethics and emotional strength

Continuous education for nurses.

4.2.2 The concept of 'health education' as 'educating individuals to promote their health'.

In that category there were sorted students' answers that regard health education as the education of the community which aims at health promotion, illness and disabilities prevention and quality of life. Some students specifically mention that health education must be a responsibility of health specialists. For example,

Learning basic principles of health promotion in the individual and common level'

Learning for health, the practices which will bring emotional and physical balance

Giving help and knowledge to younger people about health

Health culture'

Given knowledge from health professionals to the community in order to protect and promote health

Teaching people about health, prevention and self-care

Seminars and health promotion programs.

4.2.3 The concept of 'health education' as 'patients' guidance'

Nurse defined health education as the guidance and education given to a patient in order, for instance, to cooperate and follow the treatment. This way, students point out the important pedagogical role of health professionals to their patients. For example,

Teaching a patient to take care of himself'

As nurses we have a consultative role to teach the patient how to take care of himself mentally and physically

Patient's education about the care given

Those who are treated for an illness must be taught and guided about compliance to the treatment, etc. 


\subsection{The concept of 'health care'}

4.3.1 The concept of "health care" as the "social welfare about health"

Nursing students believe that health care relates to the actions being followed aiming at forming necessary policies that protect and promote health.

For example,

The sensitization of policy makers about health such as building hospitals and bringing new technologies to health care services

Health care refers to the society taking care of citizens' health, for instance by building hospitals or teaching health professionals.

4.3.2 The concept of 'health care' as 'the individuals' responsibility to promote their own health'

Moreover, nursing students' answers about the meaning of health care, reveal their opinion that health care is strongly related to the individual's effort and responsibility to acquire knowledge and take action to protect his/her physical, emotional and social health.

For example,

Health care means that someone cares for the life and happiness of those whom he loves

It means someone's effort to improve his physical and emotional well being

It is everyone's need to take care of himself, to learn about illness.

4.3.3 The concept of 'health care' as 'health promotion'

It is worth mentioning the nursing students' perception that health care is the alternative meaning of health promotion. In many of their answers they use the concepts of 'health promotion' and 'prevention' to define the concept of 'health care'. For example,

It is the given knowledge, values, ways of dealing and even materials to stay healthy

It means the well being, physically and emotionally.

4.3.4 The concept of 'health care' as the 'health professionals' effort to take care of their patient'

Nursing students answered that health care refers specifically to the services that health professionals provide to patients. For example,

Health care is the effort of nurses and doctors to improve health

For nurses it means taking care of patients

As nurses we aim at practicing methods to provide good services concerning physical health, keeping people informed and being supportive

It means all these methods specialists use to protect health

4.4 The concept of 'health ethics'

4.4.1 The concept of 'health ethics' as principles and values in nursing

Fry \& Johnstone (2005) mention that in nurse practice important ethic principles are beneficence and non harmfulness, justice, autonomy, sincerity, reliability, defence, responsibility, cooperation, care.

Students gave answers that relate health ethics to the above mentioned principles. They describe the meaning of the term "health ethics" using terms such as 'respect' to the patients and their decisions regarding their treatment, justice while practising health care, cooperation between the professionals, concern. For example,

Health ethics means to be fare and not expect the patients return

All people must be taken care of equally, despite their history

Health ethics is the fact that health professionals overcome stereotypes and prejudices, and work with patients and colleagues with no discriminations

It is the respect to the needs and beliefs of the patients regardless their convictions

To work in a way that our priority is the patient being well

To deal with the patient respecting him as a human being and giving support and care

To deny bribery and to have no racism against patients 
Cooperation, fare attitude, privacy

To take into consideration the patients' choices and decisions about their health and to follow ethic rules in decisions such as life cease.

4.4.2 The concept of 'health ethics' as a total of rules

In this category more general definitions of the concept under study were included, which gave to 'health ethics' the meaning of a total of rules that health professionals must follow in their practice and decision making. However, nursing students were not able to define which these rules are or what their content is. For example,

The term 'health ethics' means stereotypes that are followed

It is the ethical barriers in health

It is the parameters that health staff must take into consideration when providing health services and enable them to decide right and wrong

The limits a health professional must respect

4.5 The concept of 'illness'

The answers were sorted based on the meaning of 'illness' as:

Sickness

Lack of health

Malfunction

Damage, disorder, weakness, problem

The conscious someone has about his/her physical-emotional status Danger of dying

4.6 The concept of "safety in health care"

Nursing students answered that 'safety in health care' involves both health professionals and the patients - users of the health system.

The first category includes answers regarding 'who the safety in health care is given to', that is, which is the subject that enjoys safety. Two subcategories were formed: one referred to 'safety in health care that is provided to health professionals' and the other to 'safety in health care given to patients-users of the health system'.

For example, some of the students' answers are:

'Safety concerns the safety of the health care provider and the health care receiver alike'

'Safety in health care means health safety for everyone related to health care'

'Making sure that there is no danger neither for the people who provide care, nor the patients'

'Safety of the patient who receive care and the health scientists who provide care as well'

'Safe environment both for those who provide care and those who receive it'.

On the second category, the students' answers concerning the way in which, according to their point of view, the safety in health care is secured, that is which are the necessary conditions to establish the safety of health care, were classified. This category consists of sub-categories referring to:

Rules, regulations, decrees, protocols

Knowledge and qualification of the health scientists

Techniques and planning which should be used by the scientists when providing care, so as to assure safety in health care

Equipment and technical support required at health care services

Respect to the patient by keeping the medical privacy. Furthermore, in this sub-category two references were included referring to the confirmation of the patients' privacy

Safe environment, meaning conditions in general that assures safety in health care. In this sub-category students' answers which couldn't be included to the other five sub-categories were sorted.

On the third category, students' answers referring to 'which is the outcome of health care provision that proves safety' were sorted, that is, which is the proof that there is safety in health care. Sixteen references were sorted, classified into five subcategories as presented below: 
Safety means prevention of illnesses' and microorganisms'

Safety means preventing and limiting dangers to health

Safety means efficacious cure

Safety means how the patient him/her self experiences the feeling of being safe

Safety means protection from accidents in health services and safe environment in general.

4.7 The concept of 'quality in health services'

According to the literature, health care can be divided into structure, procedure and outcome, in order to study quality in the health services (Tountas, 2008). Based on the students' written texts and the content analysis of them, we classified the answers into four categories, as presented below:

A. About the concept of 'quality in health services', we sorted the students' answers that referred to 'structures'. Three sub categories belong in this category:

Education and training of health scientists. In this subcategory are included answers that refer both to scientific and technical training and guidance to follow the profession's deontology

Material, substructure, buildings

Sufficiency of health professionals.

B. In the second category, references on the procedures that assure quality in health care services were sorted. These procedures, according to the students' answers, are related to the methods used, the evaluation and research being held, the planning and time in delivering services and generally to the 'environment' in health services. For example:

'Quality includes methods, evaluation and research aiming at the improvement of health systems'

'Quality means right planning'

'Quality means to be able to engage physical and emotional health of the patient through reliable and well performed nursing care that I provide as a nurse'

'Quality in health service means good service for the patient and securing safe conditions'.

C. In the third category, answers relating to the quality in health services and the outcome of care are sorted. The outcome relates to the patients' satisfaction, the health professionals' satisfaction and the fulfillment of their needs according to the scientific knowledge, such as the time a service needs to be taken care of. For example:

'Quality has to do with good working conditions'

'Quality means that] health care is being provided with no discrimination to those who need it'

'Quality means no hospital infections, short waiting time in a hospital, satisfaction of expectations and demands'

'Providing health services focused on human and having as the most important goal to fulfill the patient's needs'

'Being quick when providing health care'.

D. In the fourth category of the meaning of 'quality in health care', we sorted the students' references that could not be included in the above three categories, as they were very general. For example:

'It is the way we provide health services'

'Quality is the best possible health services that can be provided'

'Quality means to make society sensitive about groups in need and the creation of a fare health system'

'Quality is providing health services respecting patient's personality'.

\section{Discussion}

Reviewing the concepts of 'health', 'health care', 'health education', 'health ethics', 'illness', 'safety in health care' and 'quality in health care' it is obvious that they are multidimensional in view of different sciences. According to that:

$\Rightarrow$ Health is a wide field of discussion. Society's needs and requirements have grown and the concept of health is strongly related to individual and civil rights. Moreover, health includes four distinctive concepts (the four "c") (Liaropoulos, 2007): (a) Care, (b) Cure, (c) Control, and (d) Community. 
The concept of Care is related to the holistic treat of health in the individual or group level and care is headed to the community as whole. The true meaning of health care is defined by the aims of the health system, which are prevention, cure and dealing with the consequences of illness. The concept of Health Care has often been related to Nursing Science. As Leninger underlines in Sapountzi-Krepia D., Krepia V. (2005), 'care is not only related to nursing, but it is also a universal phenomenon that appears with differences in every human culture'.

$\Rightarrow$ Health education is an education trend that, aiming at the interests and needs of young people, uses methods and pedagogical techniques in order to empower health choices and positive life attitudes. Moreover, health education aims at the processing of experiences and emotions, the empowerment of self-esteem and trust, as well as the development of social relations through the cultivation of social skills (Makris, 1992).

$\Rightarrow$ Health ethics refer to the ethical dimensions of actions and choices of health professionals in their daily practice and aim at the sensitization of professional on ethical decision-making and moral acts. Such issues include dimensions of deontology with serious medical, moral, social, legal, economic even religious extensions and being the subject of study of bioethics (Downie \& Calman, 1997).

$\Rightarrow$ The terms 'illness', 'disease', 'sickness'describe the health status of a person in a negative way; alternatively (but wrongly), these terms are often used to describe a real, negative situation according to the way it appears and the consequences on a person's body and function. Illness has the consequence of clinical symptoms or laboratory findings and the intervention required from the health system is treatment (Liaropoulos, 2007).

$\Rightarrow$ Safety is one of the basic human needs. Safety must be taken into consideration throughout health care and constitutes a major responsibility for health professionals. Safety issues involve every health care provider and all age groups (Taylor et al., 2006). Knowledge, detecting and setting right every dangerous situation in health structures consist a top priority for the individual and the society.

$\Rightarrow$ Quality in health services: according to the Organisation for Economic Cooperation and Development (2004), it is defined as 'the extent to which health services manage to raise the possibility for health to be reached and they are consistent in modern science' (Liaropoulos, 2007). According to Donabedian, quality in health services consists in technical quality and interpersonal quality. Technical quality depends on the knowledge and critical thinking used to select the appropriate health care strategies and the ability to follow these strategies in practice. Handling the interpersonal relations is the second component for quality in health care. Through interpersonal reconciliation, the patient gives the important information regarding his desires, which is necessary to select the appropriate care methods (Liaropoulos, 2007).

Finally, a critical examination of the content of these concepts would guide us to:

The need to create cultural structures and procedures to continuous education for health promotion (Gasey, 2007), and

The importance of the quality in health promotion, which defines the human being in multiple ways (Bakalis \&Theodorakopoulou, 2006).

Nursing students' and future health professionals' answers on the meaning of basic concepts, such as 'illness', 'safety' and 'quality' in health care, show how sensitive they are regarding the related subjects. The result of the students' opinion reveals the way they conceive their professional role and their education (Mlinar, 2010) their attitude in real working conditions and, therefore, the quality of the services they provide and how well prepared they are to demand and to put in a claim for the quality in health care.

Healthcare quality continues to be a subject of intense criticism and debate. Although quality nursing care is vital to patient outcomes and safety, meaningful improvements have been disturbingly slow. Analysis of quality care literature reveals that practising nurses are rarely involved in developing or defining improvement programs for quality nursing care. Therefore, two major study premises were that quality nursing care must be meaningful and relevant to nurses and that uncovering their meaning of quality nursing care could facilitate more effective improvement approaches. Nurse educators could modify education curricula to model and teach students the intrinsic qualities identified within these meanings of quality nursing care (Burhans \& Alligood, 2010; Williams $\&$ Taylor, 2008). Nursing knowledge of the meaning of quality nursing care for practising nurses has potential to refine the discipline and facilitate practice changes, driving improvements in quality care and patient outcomes (Pope, van Royen \& Baker, 2005).

While nursing science needs to create learning environments that empower the dynamics of holistic care, support multi-dimensional education and underline the importance of professionals' ethical approach, the present study reveals the nurse students' sensitization and knowledge over major concepts regarding health (Parsons, Barker \& Armstrong, 2001). 


\section{Conclusions}

The analysis revealed that nurse students relate health to the physical and emotional status of a person, regard health education as the health professionals' training, but also as a person's culture and attitude regarding health. They also believe that health care is a responsibility for individuals as well as the community as a whole and relate the concept of ethics to respecting the human value. Further empirical research could aim to replicate or contradict these findings, using a larger sample and recruiting more university departments.

\section{References}

Burhans LM, Alligood M.R. (2010). Quality nursing care in the words of nurses. Journal of Advanced Nursing, 66(8):1689-1697.

Downie R S, Calman KC. (1997). Health respect. Ethics in health care. Greek for Ethics and Deontology. Athens: Litsas Medical (Greek).

Fry S, Johnstone MJ. (2005). Ethics in Nursing Practice. A Guide to Ethical Decisions. Lemonidou, Ch. (Translation- Supervision). International Nurse Council. Athens: Paschalidis. (Greek).

Gasey D. (2007). Issues in clinical nursing. Nurse's perceptions, understanding and experience of health promotion. Journal of Clinical Nursing, 16: 1039-1049.

Graneheim UH, Lundman B. (2004). Qualitative content analysis in nursing research: concepts, procedures and measures to achieve trustworthiness. Nurse Education Today, 24: 105-112.

Ioannidi V. (2008). Ethics and educations for health. Basic issues on Bioethics, Health Education and Promotion. Series: Health Library, no 1. Supervision of the series" P. Baltopoulos. Athens: Paschalidis. (Greek).

Kotrotsiou St, Paralikas Th, Papathanasiou I, Lachana E, Kiparisi G, Rizoulis A. (2008). The concept of care. Rostrum of Asclepius, 1(7): 57-71 (Greek).

Liaropoulos L. (2007). Health Services \& Systems Management. (vol A) Athens: Vita. (Greek).

Makris C. (1992). Health Education in the European Union. Logos \& Praxi, 50: 23-39 (Greek).

Mlinar S. (2010). First- and third-year student nurses' perceptions of caring behaviours. Nursing Ethics, 17(4): 491-500.

Mpakalis N, Theodorakopoulou G. (2006). Quality. Important parameter in Nursing. Nursing, 45(3): 352-358 (Greek).

Parsons S, Barker PJ, Armstrong AE. (2001). The Teaching of Health Care Ethics to Students of Nursing in the UK: a pilot study. Nursing Ethics, 8: 45-56.

Pope C, van Royen P, Baker R. (2005). Qualitative methods in research on healthcare quality. Quality and Safety in Healthcare, 11:148-152.

Ryan G W, Bernard RH. (2000). Data management and analysis methods. In: Denzin, N., Lincoln, Y., (Eds.), Handbook of qualitative research. $2^{\text {nd }}$ ed. Thousand Oaks, CA: Sage. p. 769-802.

Sapountzi - Krepia D, Krepia V. (2005). Philosophical aspect of nurse care. Nursing, 44 (2): 178-185.

Sparos L. (2001). Meta-epidemiology. Athens: Vita. (Greek).

Taylor C, Lillis C, LeMone P. (2006). Basic Principles of Nursing Science and Art of Nursing Care. (Lemonidou C, Patiraki-Kourmpani E. (vol III). Athens: Paschalidis. (Greek).

Tountas I. (2008). Health Services. Odysseas/Nea Ygeia: Athens. (Greek).

Vamvoukas M. (1088). Introduction to psychosocial research and methodology. Athens: Grigoris. (Greek).

Williams A, Taylor C. (2008). An investigation of nurse educator's perceptions and experiences of undertaking clinical practice. Nurse Education Today, 28: 899- 908. 